Running Head: COPING CONCORDANCE IN COUPLES

Coping Concordance in Couples 


\begin{abstract}
Romantic partners' daily coping behavior can be viewed as a context for one's own coping. Across 3 studies we found that individuals were more likely to cope actively, seek support, or use alcohol, food, and drugs as means of coping when they perceived their partners doing so on a given occasion (Study 1, a cross-sectional study), and when their partners reported using these strategies (Studies 2 and 3, longitudinal couples studies). These effects were evident regardless of whether or not one partner was dealing with an acute stressor (Study 2), or if both partners were dealing with day-to-day hassles (Study 3). Although these patterns are correlational, they raise important questions about how individuals choose to cope with acute stressors.
\end{abstract}

Keywords: context, stress, regulation, daily diary methods, dyadic data analysis 


\section{Coping Concordance in Couples}

Coping is widely considered to moderate the effects of stress (e.g., Bargiel-Matusiewicz \& Hatim, 2015; Bigatti, Brown, Steiner, \& Miller, 2011; Folkman \& Lazarus, 1988; Folkman, \& Moskowitz, 2000; Sirois, Molnar, \& Hirsch, 2015). Although the literature is mixed regarding which coping strategies are effective for alleviating distress, certain strategies (e.g., problemfocused and active coping) are regarded as more effective than others (e.g., avoidant coping such as denial) at managing stressors. Given that using certain coping strategies may have the potential to alleviate or exacerbate individual distress, it is crucial to better understand how couples use specific coping strategies.

The social context in which coping occurs is important to consider. For instance, it is well established that close relationships can greatly impact how effectively individuals are able to deal with distress (for a review see Eckenrode, 2013). We propose that one important factor in how individuals cope with distress may be the coping efforts of romantic partners. Specifically, we posit that individuals are more likely to enact certain coping behaviors if their partners are engaging in those behaviors as well. We propose that this occurs in a process that we refer to as coping concordance that tracks time-varying coping similarity above and beyond similarity in general coping tendencies or styles.

\section{Coping Behavior in Couples}

Much of the coping research has drawn on the Lazarus and Folkman (1984) transactional model of stress - a view that the coping process is a dynamic one and highly affected by social factors. However, relatively little research has explored how one romantic partner's coping strategy use is related to one's own coping behavior. The social context of coping matters: One's stressors and distress extend beyond the individual's own experience and affect close others 
(e.g., Eckenrode, 2013). Hence, the coping strategies an individual enacts tend to affect not only the self, but close others as well.

More fundamental than the dyadic effects of coping is the question of whether or not a partner's coping behavior affects the kinds of coping behaviors one enacts as well. Some older research has found evidence of similarity in coping strategy use between romantic partners (BenZur, Gilbar, \& Lev, 2001; Giunta \& Compas, 1993; Revenson, 2003) in their overall tendency to use one kind of coping or another. All of these studies defined similarity as a correlation between tendencies to use certain coping strategies, and did not specifically address day-to-day similarity that may arise above and beyond similarity on average. Evidence also suggests that there is convergence of strategy use over time in couples (Holahan et al., 2007), but even this longitudinal study used measures of average coping style - or dispositional coping - over the previous year, rather than a specific coping association that may occur day to day.

Similarity at the dispositional level may be due to assortative mating or it may reflect gradual growth in similarity over time (Holahan et al., 2007). If a person selects a partner who is especially similar to himself or herself, the couple may enter the relationship using similar coping strategies. Such similarity may not reflect a process of interlinked coping behaviors in the couple from day to day, but rather a selection process at relationship initiation - that is, that individuals tend to choose partners with similar backgrounds and attitudes (for a review see Watson et al., 2004). Because personality is associated with using certain coping strategies (Carver \& Connor-Smith, 2010), it is feasible that individuals who choose partners with similar personality traits may already use similar coping strategies. For instance, partners who are especially optimistic may be more prone to engage in active, problem-focused coping (Solberg Nes \& Segerstrom, 2008) both before and after entering the relationship, independent of the 
other partner's use of active coping. Due to this selection, partners may appear particularly similar in use of a strategy from day to day, yet this similarity reflects a stable characteristic of the couple instead of a day-to-day dynamic process. Similarity in average coping strategy use, however, is quite different from similarity that emerges as a result of both partners engaging in the same coping behavior on specific days or modeling partner's same-day coping behaviors. Indeed, it has been shown that dispositional coping correlates only weakly with coping at specific times and in specific contexts (e.g., Todd, Tennen, Carney, Armeli, \& Affleck, 2004).

\section{The Present Research}

The goal of the present research is to examine the degree of daily coping concordance between romantic partners over time while taking into account general coping style similarity across three studies. To our knowledge, no research has examined coping concordance that occurs day to day while also taking into account both one's own and one's partner's general coping styles. The few longitudinal studies that might have been able to capture change in coping behaviors over time have been either very short or have measured few time points (e.g., Holahan et al., 2007). Intensive longitudinal designs with couples over several weeks are ideal to determine the actual process in daily life because they allow us to examine coping similarity above and beyond one's overall tendencies to cope in a certain way.

In the present research we explicitly distinguish between coping concordance that arises from similarity of dispositional tendencies and concordance that arises from specific daily processes, while also enumerating possible mechanisms that may facilitate coping concordance such as direct encouragement and modeling of coping. We expect that daily coping concordance will be specific to the type of coping behavior initiated by the partner. Although there are many ways to categorize coping behavior types (e.g. Skinner, Edge, Altman, \& Sherwood, 2003; 
Carver \& Connor-Smith, 2010; Zimmer-Gembeck \& STkinner, 2011), we focused on three groupings of behaviors that we believed would be sufficient to show the coping concordance specificity effect: Active-problem focused coping, support-seeking, and food/alcohol/substance use. Active-problem focused coping represents engagement with the stressor and has the potential of reducing its magnitude. Support seeking may reflect either engagement or disengagement, but certainly involves aspects of emotional coping. Lastly, food/alcohol/substance use reflects disengagement coping that might temporarily reduce the emotional reaction to the stressor, but leaves the magnitude of the stressor unaffected.

We report the results of three studies that estimate the degree of concordance between an individual's coping behavior and the partner's coping behavior on a given day, while taking into account general coping style similarity. In Study 1, using a cross-sectional survey, we sought to investigate the extent to which individuals notice their partner's coping behaviors and whether or not such awareness of a partner's coping may affect the individual's own coping. We examined the association between an individual's reported own coping behaviors in response to the most demanding aspect of one's day and perceptions of his or her partners' coping behaviors that day.

In Study 2 we sought to extend the findings in Study 1 by establishing the coping concordance effect of daily coping in secondary analysis of a longitudinal diary study (Shrout et al., 2010). Specifically, in this analysis we examined whether or not both partners actually report using the same coping strategies on a given day. Study 2 participants were cohabiting heterosexual couples in which one member of the couple was preparing for the state bar examination in the coming month, while their non-examinee partners contended with daily life hassles. Hence, this sample exhibited a role differential within the couple as a function of the stressor. We predicted that regardless of role, the concordance effect between partners would 
emerge for active coping and alcohol, food, and drug use across the entire month leading up to the bar exam. However, given the increase in distress for examinees as the exam approaches, we expected the association between partners' support seeking to diminish or reverse (become negative) the week prior to the exam, when examinees are most stressed and engaging in more emotion-focused coping (i.e., support seeking and alcohol, food, and drug use, lida et al., 2017). We also examined whether or not time spent together on a given day moderated partner's same day effect on coping, reasoning that more exposure to the partner's coping would increase the tendency to choose the same coping strategies as the partner.

In Study 3, we aimed to replicate the concordance effect of daily coping from Study 2, and extend it to a sample of couples without the examinee-non-examinee role differential who completed a daily diary protocol that was very similar to that of Study 2 .

\section{Study 1}

Before examining daily coping concordance, we first wanted to establish whether or not partners notice their mate's coping as well as whether or not their mates encourage them to cope in a certain way in a cross-sectional study. If a partner advises one's mate to use a particular coping strategy, he or she may be more likely to use that strategy. This type of coordination is what we assume occurs when partners engage in dyadic coping - that is, when partners communicate stress to each other and focus on coordinating coping efforts (e.g., Bodenmann, 1997). Hence, the association between partners' coping behavior may depend in part on a partner's explicit encouragement which we examined in addition to perceived partner's coping. Participants in Study 1 completed a questionnaire in which they reported the kinds of behaviors they enacted that day to cope with the most demanding aspect of their day. They also reported whether or not their partners experienced a particularly stressful event that day and how 
they coped with it if they had. In addition, participants reported how much time they spent with their partners that day - a factor that may moderate coping similarity between partners.

We predicted that participants would be more likely to use a specific coping strategy type (active coping, support seeking, and alcohol, food, and drug use), if they perceived their partners doing so that day and if their partners encouraged use of that strategy.

\section{Method}

\section{Participants and Design}

Participants. One hundred and twenty three participants were recruited via Amazon's Mechanical Turk and were compensated $\$ .75$ for their participation. We excluded 2 participants who were not in a romantic relationship, 5 who were in long distance relationships, and 14 participants who did not follow directions when completing the study. Our final sample consisted of 102 participants $($ males $=44$, females $=57$, transgender $=1$ ). Participants were 33.76 years old on average $(S D=10.52)$. They identified themselves as $90.2 \%$ Caucasian, $6.9 \%$ Latino, $3.9 \%$ Asian, 3.9\% Black, and 2.0\% American Indian. Participants also identified themselves as $94.1 \%$ heterosexual, $1.0 \%$ as lesbian, $1.0 \%$ as gay, and $3.9 \%$ as bisexual. Most participants were cohabiting with their partners $(83 \%)$ and $54.9 \%$ were married.

Procedure. Participants on Amazon's Mechanical Turk were invited to take part in a study assessing everyday behaviors of individuals in close relationships. After consenting to the study, they completed an online questionnaire by answering various questions about the activities they engaged in the past 24 hours.

\section{Measures}

Self-reported coping behaviors. To examine different coping strategy types, we asked participants to name the most demanding event they experienced in the last 24 hours. Then, from 
a list of 21 behaviors, some of which were adapted from the COPE (Carver, Scheier \& Weintraub, 1989), they clicked "yes" or "no" to indicate which of the behaviors they used to cope with the demanding event. For a discussion of coping strategy typology see the online supplement "Coping Typology."

We took the average of the specific binary coping strategies to create active coping, support seeking, and alcohol, food, and drug use composites. Active coping consisted of 5 strategies that were aimed at dealing with the specific stressor directly, including "made a plan to fix a problem," "persevered," "did something to deal with the problem," "accepted the situation," and "looked at the problem positively." Support seeking - a more social coping strategy that should emerge readily in romantic relationships - consisted of "talked to romantic partner," "talked to family member or friend," and "vented about some issue." The alcohol, food, and drug composite consisted of "had one or more alcoholic beverages," "smoked," "used recreational drugs," "used prescription drugs designed to relax, induce sleep, or reduce stress," and "indulged in a special food" - strategies that are aimed at dealing with the emotional experience associated with a stressor. These composites ranged from 0.0 if participants had used none of the coping strategies in a given type to 1.0 if they had used all of the strategies in a type. Means, standard deviations, and correlations for these items are presented in the online supplement "Coping Typology."

Participants were then presented with the same list of coping behaviors as before and asked to indicate which they performed because their partners encouraged them to do so. Their binary responses for each of the coping strategies were averaged to produce a measure that was comparable to the reports of coping behavior.

Time spent together. Participants answered the following question to assess how much 
time they spent with their partners that day: "During your waking hours how much time did you spend with your partner in the last 24 hours, physically in the same room?"

Perceived partner's coping behavior. Participants were also asked whether or not their partners had experienced a particularly stressful event that day. They were then presented with the same list of coping behaviors as before and asked to indicate which behaviors they perceived their partners engaging in to cope with the stressor.

\section{Results}

\section{Coping Behaviors and Descriptive Statistics}

Means and correlations for participants' and perceived partners' use of coping strategy types are presented in Table 1. Participants used active coping strategies the most (active coping vs. support seeking $t(101)=1.94, p=.056$; active coping vs. alcohol, food, and drug use $t(101)=$ 19.93, $p<.001)$. Support seeking was also used more frequently than alcohol, food, and drug $($ AFD) use $(t(101)=11.16, p<.001)$. Partners were perceived as seeking support more frequently than coping actively $(\mathrm{t}(74)=2.57, p=.012)$, and using alcohol, food, and drugs the least (active coping vs. AFD $t(74)=7.40, p<.001$; support seeking vs. AFD $t(74)=9.16, p<$ $.001)$.

\section{Own Coping Strategy Use}

To test the association of perceived partner's coping and partner's encouragement to cope with one's own coping, we performed a series of multiple regression analyses with active coping, support seeking and AFD use as outcomes in SPSS (v20.0, 2011). All predictor variables in these and subsequent analyses were centered at their respective means.

The perception that one's partner was actively coping was associated with one's own active coping $(\beta=.40, t(72)=3.84, p<.001)$, after adjusting for partner's encouragement to 
cope actively, which also had a unique association with one's coping $(\beta=.26 t(72)=2.51, p=$ .014). The magnitude of the effects of partner's coping and partner's encouragement was comparable for active coping $(\beta=.40$ vs. $\beta=.26, t(72)=.18, p=.861)$.

A similar pattern of results emerged for support seeking. Specifically, if partners were perceived to seek support that day, participants were also more likely to seek support even after adjusting for partner's encouragement $(\beta=.23, t(72)=2.08, p=.042)$; partner's encouragement itself had a significant unique effect $(\beta=.31, t(72)=2.84, p=.006)$. Although the size of the effect of encouragement appeared larger than the partner's coping behavior, it was not significantly different, $(t(72)=1.33, p=.188))$.

Perceived partner's AFD use was marginally associated with one's own AFD coping $(\beta=$ $.20, t(72)=1.71, p=.091)$, after we adjusted for partner's encouragement to cope using AFD. Partner's encouragement to cope using AFD, however, was significantly associated with one's own AFD use, $(\beta=.40 t(72)=3.51, p=.001)$ with an effect size that was marginally different from the marginal effect for partner's AFD coping $(t(72)=1.98, p=.051))$.

\section{Coping Association Specificity}

We predicted that the effect of perceived partner's coping on one's own coping would be evident primarily for the same strategy types (i.e., coping concordance). However, one can see from the correlations in Table 1 that, for example, perceived participant's active coping is significantly associated with perceived partner's support seeking. To examine the specificity hypothesis, we regressed each participant's coping strategy on all three perceived partner coping variables and a) examined the patterns of tests of unique effects, and b) tested whether the effect for the specific perceived partner coping was significantly larger than the two non-specific unique effects. 
We found mixed effects for the specificity hypothesis. Own active coping was still significantly predicted by partner's active coping after adjusting for partner's use of the other strategies (support seeking and AFD use), but perceived partner's active coping was not a significantly stronger predictor than the two other strategies. Own support seeking was no longer significantly predicted by perceived partner's support seeking when adjusting for perceived partner's use of the other two strategies (active coping and AFD use). Like for active coping, own AFD use was still significantly predicted by perceived partner's AFD use, after adjusting for perceived partner's use of the other two strategies (active coping and support seeking). In this case, perceived partner's AFD use was a significantly stronger predictor of participants' AFD use than the two other strategies. Detailed results are available in the online supplement, Table 1.1.

\section{Gender Effects}

Even though we did not expect to see gender differences in coping concordance between partners, we tested this directly. None of the coping strategies that were perceived to be used by the partner interacted with the participant's gender to predict his or her coping. A discussion of gender effects for Studies 1, 2, and 3, can be found in "Gender Effects" of the online supplement.

\section{Process Mechanisms}

We wondered if the pattern of findings might be moderated by time spent with partner. We examined this by including this variable in the regression models reported above, both as main effects and as interactions with partner's coping. Time spent with partner was not significantly related to any of the three participant coping behaviors, and including this variable in the model as a main effect and interaction did not change the pattern of significance of findings. There was no evidence of an interaction between time spent together and perceived 
partner's coping. Results are available in the online supplement, Table 1.2.

\section{Discussion}

As predicted, participants who reported using active coping, support seeking, and AFD as coping strategies were more likely to report that their partners used the same strategies. Partner's encouragement to cope in these different ways also independently predicted participants' use of the specific strategy types. The independent effects of perceived partner's coping and partner's encouragement to cope were comparable for active coping, and support seeking, but encouragement to use alcohol, food, and drugs was a stronger predictor of one's use of this strategy than perceived partner's use of this strategy. These results suggest that for active coping and support seeking there seems to be a balance between more directive efforts of the partner as well as the perception of partner's use of the same strategy.

We did not find evidence that time spent together impacted the association between one's own and perceived partner's coping. The results for partner's coping association specificity were also mixed. For own active coping and support seeking, perceived partner's use of the same strategy was not a stronger predictor than perceived partner's use of the other coping strategies, indicating no specificity. For own alcohol, food, and drug use, the partner's use of the same strategy was a stronger predictor than the other two coping strategies, suggesting that the effect of perceived partner's coping is specific for this strategy.

Study 1 served to show preliminary evidence that individuals do in fact notice their partners coping behaviors, and that perceptions of a partner's coping is positively correlated with one's own coping strategy use. Nevertheless, it was limited in a number of ways. First, the sample included only one member of the couple so it was not possible to measure partners' coping behaviors directly and compare both partners' perspectives. Surely some coping efforts 
escape the partner's notice. It is likely then that Study 1 missed a substantial portion of coping that may not have been consciously noticed by participants. Similarly, one can argue that the apparent association between partners' coping may arise as a function of perceived similarity, such that participants who used certain coping strategies assumed that their partners also used the same strategies - that is, that they projected their own coping behaviors onto their partners (Kenny \& Acitelli, 2001). The results of Study 1 shed light only on the correlation between individuals' perceptions of partner's coping behaviors and their own coping strategy use and thus do not rule out this alternative explanation. In addition, we assessed coping behaviors in response to a specific stressor at only one time point and we were not able to distinguish participants' and partners' average use of a coping strategy from a stressor-specific coping response. In the next study, we sought to address these limitations by examining coping concordance in both members of a couple and over time.

\section{Study 2}

Study 2 made use of longitudinal diary data from a study of support processes in couples experiencing stress (Shrout et al., 2010). Through secondary analysis of these data we sought to extend the findings in Study 1 by establishing the coping concordance effect - a correlation between partners' coping behavior on a given day - while also taking into account general coping styles. Specifically, we examined the coping concordance process over time, accounting for couples' average coping tendencies (i.e., coping styles) as well as daily variations in coping. We also sought to extend our findings by demonstrating them in a sample of couples in which one of the partners was experiencing an acute stressor (preparing for a state bar exam) while the other was dealing with stresses of everyday life. Participants completed an initial background questionnaire and then filled out morning and nightly diaries for 33 days leading up to the bar 
exam $^{1}$. In the nightly diaries they indicated how they coped with challenges during their day.

We predicted that participants would be more likely to use a particular coping strategy on a given day if their partners used the same strategy regardless of their own and their partner's average use of that strategy over the course of the study, and regardless of their own use of that strategy the previous day. We examined time progression over the course of the study as a proxy for demand of the stressor. Specifically, we hypothesized that as demand of the stressor increased for examinees - that is, the bar exam drew near - the coping concordance effect for support seeking would decrease. We predicted that partners of examinees - the non-examinees would be less likely to seek support in response to examinee's support seeking on the week prior to the bar examination, a period of time in the study shown to be qualitatively different in terms of stress and coping strategy use (Iida et al., 2017). We otherwise did not expect to see differences in coping concordance based on role, although the role differential in this sample of couples may contribute to potentially different magnitudes of concordance effects for examinees and non-examinees. Even though we did not find evidence that time spent together moderated the effect of perceived partner's coping on one's own coping in Study 1, we re-examined whether or not this variable moderated coping concordance on a given day.

\section{Method}

\section{Participants and Design}

Participants. Law students who were currently preparing for the state bar exam were recruited for the study via outreach to more than 100 law schools. To be eligible to participate in the research, examinees had to speak fluent English, and be in romantic relationships with partners whom they were cohabitating for more than 6 months. Only those whose romantic partners agreed to participate and were not also studying for the bar exam were eligible. 
All examinees took the bar exam in mid-July of 2001, 2002 or 2003. Of the 100 law schools in the United States that were contacted, 34 agreed to participate in research that was examining coping behaviors of students in romantic relationships (Gleason, Iida, Shrout \& Bolger, 2008). Representatives of these law schools advertised the study via email and by distributing flyers. Individuals interested in the study contacted the researchers directly.

The final sample of eligible participants who agreed to take part in the study consisted of 306 heterosexual couples, but complete data for both members of the couple were available for 294. On average, examinees were 29.6 years $(S D=6.4$ years $)$ and non-examinees were 29.8 years $(S D=7.8)$. Ethnically examinees identified themselves as $77.7 \%$ Caucasian, $4.5 \%$ Latino, 5.2\% East Asian, 1.9\% other Asian, .9\% Black, .8\% American Indian. Additionally 3.3\% identified themselves as "other" and $1.1 \%$ as multi-ethnic. Non-examinees identified themselves as $81.0 \%$ Caucasian, $4.3 \%$ Latino, $4.6 \%$ East Asian, .9\% other Asian, $3.2 \%$ Black, .7\% American Indian, 1.9\%“other," and .6\% multi-ethnic. Sixty-four percent of the couples were married.

Procedure. Two months prior to the bar exam participants received a background questionnaire assessing demographic, personality, and various relationship measures. Then, for 34 days leading up to the exam and 10 days after the exam, both examinees and their partners filled out daily diary reports in the morning and at night. The daily diaries were designed to take approximately 5 minutes to complete and included questions regarding mood, support provided and received during the day, and daily troubles and stressors, as well as behaviors enacted to cope with the stressors encountered during the day.

\section{Measures}

Time spent together. In the evening diaries participants indicated how much of their 
waking time they spent with their partner that day ("Of the hours you were awake approximately how many of them did you spend with your partner?'). Because examinees' and non-examinees' reports were highly correlated $(r=.77)$, we averaged them together.

Daily coping. In the nightly diaries, participants were presented with 12 coping behaviors adapted from the COPE (Carver, Scheier \& Weintraub, 1989) as a checklist, and indicated which behaviors they used to deal with the most demanding or difficult aspect of their day (i.e., they responded how they dealt with a stressor every day during the study). We were particularly interested in the following coping strategy types: active coping (mean of items "accepted the situation," "did something to deal with problem," "persevered," "planned," and "looked at problem more positively"), alcohol, drug, and food (AFD) use (“ate/smoked/drank/used drugs"), and support seeking (mean of items "sought emotional support," "sought practical support," and "let out negative feelings"). All items were coded 0 for "no" and 1 for "yes."

Diary days. The specific day in the daily diary portion of the study was used as a measure of time progression, and is represented as a continuous variable. Only days $2^{2}$ thru $34-$ the days before the bar exam - were analyzed. We centered the variable on day 2 , so that it ranged from 0 to 32. A weekend day variable was also created with days that fell on the weekend coded as " 1 " and weekdays coded as " 0. ."

\section{Results}

\section{Coping Strategy Use Levels and Trajectories}

The means, standard deviations, and correlations for active coping, support seeking, and AFD use are presented in Table 3. Both examinees and non-examinees used active coping the most, and used AFD the least. On average, examinees used active coping, support seeking, and AFD more frequently than did non-examinees (active coping $t(293)=3.83, p<.001$; support 
seeking $t(293)=2.52, p=.012$; AFD $t(293)=3.83, p<.001)$. The patterns of coping strategy use over days before the exam $(2-34)$ were somewhat different for examinees and nonexaminees, especially for support seeking behavior. Whereas examinees appeared to use support seeking more as the exam neared, non-examinees used this coping strategy less as the exam drew closer in time. Trajectories of coping behaviors before the exam for examinees and nonexaminees can be found in the online supplement, "Study 2 Coping Trajectories."

\section{Coping Concordance}

To assess coping concordance in couples we used multilevel models (PROC MIXED procedure in SAS, v9.2.3, 1997) for the continuous outcomes of active coping and support coping and generalized multilevel models (GLIMMIX procedure in SAS, v9.2.3, 1997) for the binary outcome of AFD use. We estimated 6 models, half of which treated the examinee as the actor and half the non-examinee as actor; each of the three coping strategy types were modeled separately ${ }^{3}$.

The following level 1 equation was used to assess an actor's use of a particular strategy on a given day as a function of the partner's use and the actor's use on the previous day:

$$
A_{i t}=b_{0 i}+b_{1 i}\left(A_{i t-1}-A_{i} \cdot\right)+b_{2 i}\left(P_{i t}-P_{i} \cdot\right)+b_{3} D_{i t}+e_{i t},
$$

where $A_{i t}$ is the use of a particular strategy for the actor $i$ (examinee or non-examinee) on day $t$; $A_{i}$. is the actor's mean use of that particular coping strategy over 33 days; $A_{i t-1}-A_{i}$. is the actor's own person-centered use of the coping strategy on the previous day (day $t-1) ; P_{i t}-P_{i}$. is the person-centered partner's use of a coping strategy on day $t$ for examinees or non-examinees; $D_{i t}$ is the time progression through the study on day $t$ centered on day 2 , the starting day of the days analyzed for examinees and non-examinees; $e_{i t}$ is the residual of actor $i$ on day $t$. The coefficient $\mathrm{b}_{0 \mathrm{i}}$ represents the intercept of a particular coping strategy's use for actor $i$ when all independent 
variables are at their average on day 2 . The coefficient $b_{1 \mathrm{i}}$ represents the effect of the individual's own person-centered coping on day $t-1$ on his or her coping on day $t$. The coefficient $b_{2 \mathrm{i}}$ represents the effects of partner's person-centered coping on day $t$ on the actor's coping. The coefficient $b_{3}$ is the effect of time in days on the actor's use of a particular coping strategy. We did not include partners' coping on day $t-1$ as a predictor of actor's coping because we reasoned that the association between partners' coping behavior should be restricted to the same day. Since partners may engage in certain coping behaviors together (e.g., eating, drinking) a correlation between their coping should be evident on the same day only.

Each of the coefficients in Equation 1 that contains a subscript for actor $i$ is elaborated with level 2 equations. The model for the intercept $b_{0 i}$ is shown in Equation 2.

$$
b_{0 i}=\gamma_{00}+\gamma_{01}\left(\mathrm{P}_{i}-\mathrm{P} . .\right)+u_{0 i}
$$

The first term $\left(\gamma_{00}\right)$ is the overall mean of coping after adjusting for the day, lagged daily coping, and partner's coping. The second term $\left(\gamma_{01}\right)$ is the slope relating an actor's average level of coping to the partner's average level of coping over all days. This reflects dispositional coping similarity of the actor and partner. The last term in equation $2\left(u_{0 i}\right)$ is the part of $b_{0 i}$ that is not explained by the partner's dispositional coping. This is called the random effect for the intercept, and its variation is estimated rather than its specific value.

The equation for the actor's own lagged day effect is written as the simple sum of the mean lagged effect $\left(\gamma_{10}\right)$ and the random effect associated with actor $i$.

$$
b_{1 i}=\gamma_{10}+u_{1 i}
$$

The level 2 equation for the partner's daily effect (i.e., coping concordance effect) on actor's daily coping is

$$
b_{2 i}=\gamma_{20}+u_{2 i}
$$


By including effects of dispositional as well as unique daily behavior in the model, we were able to distinguish coping concordance on a daily level from concordance that arises from similarity in the usual levels of a certain coping strategy. The partner's coping effect denoted as $\gamma_{20}$, is the effect of a partner's use of a coping strategy on the individual's use of that strategy on the same day, adjusting for the tendency of both actor and partner to use that strategy across all days.

In addition, we adjusted for the possible correlation of residuals from one day to the next for the two continuous outcomes, active coping and support seeking. A Toeplitz covariance structure (restricted to 8 bands) was used to account for residual correlation; this proved to be a better fitting model than one without such an adjustment ${ }^{4}$.

Because we ran many models, the full models are presented in the online supplement, Tables 2.1 - 2.6. In Table 4 we summarize the results by presenting partner's same day and average coping fixed effects as well as the random effects for same day coping, which is the variance of the $u$ terms in equation 4 .

\section{Fixed Effects: Average Regression Effects}

Partner's same day coping effects. Partner's use of active coping, support seeking, and AFD was associated with the actor's (both examinees and non-examinees) use of each coping strategy on the same day as shown in Table 4. Examinees were more likely to use active coping on days when their partners used active coping to a greater extent than they typically do $\left(\gamma_{20}\right.$ Exam. active coping $=.053, p<.001)$ such that for every unit increase in non-examinees' use of active coping, examinees' use of active coping increased by .053 units that same day. Likewise, examinees were more likely to seek support if non-examinees sought more support on a given day than they typically do, $\left(\gamma_{20}\right.$ Exam. support seek $\left.=.046, p<.001\right)$. Finally, examinees were more 
likely to use AFD if non-examinees used AFD on a given day (logistic $\gamma_{20}$ Exam. AFD use OR $=1.46$, $p=.015)$. That is, examinees were 1.46 times more likely to use AFD on a given day if nonexaminees used this coping strategy.

A similar pattern of results indicating coping concordance emerged for non-examinees as well. Non-examinee's were more likely to use active coping on a given day if examinees used this strategy $\left(\gamma_{20}\right.$ Non-exam. active coping $\left.=.056, p<.001\right)$. Non-examinees were also significantly more likely to seek support $\left(\gamma_{20}\right.$ Non-exam. support seek $\left.=.037, p=.002\right)$ and use AFD for coping $\left(\operatorname{logistic} \gamma_{20}\right.$ Non-exam. AFD use $\mathrm{OR}=1.43, p=.020$ ) if examinees did so on a given day.

We also examined partner's same day effect on one's own coping without adjusting for one's own lagged day coping. A partner's use of active coping, support seeking, and AFD use continued to significantly predict both examinee's and non-examinee's use of the same strategy when we did not adjust for one's own coping the previous day. Results can be found in the online supplement, Table 2.7 .

Dispositional (average) coping effects. One's partner's general tendency to use a particular coping strategy was associated with increased use of that specific strategy for examinees and non-examinees (see Table 4). A non-examinee's tendency to cope actively was associated with examinee's active coping $\left(\gamma_{01}\right.$ Exam. active coping $\left.=.172, p=.007\right)$ and this was also true for support seeking $\left(\gamma_{01}\right.$ Exam. support seek $\left.=.165, p=.017\right)$. We found the same pattern of results for examinee's use of AFD (logistic $\gamma_{01}$ Exam. AFD use $\left.\mathrm{OR}=8.08, p=.003\right)$. Likewise, an examinee's general tendency to cope actively was associated with increased active coping for non-examinees $\left(\gamma_{01}\right.$ Non-exam. active. coping $\left.=.154, p=.004\right)$. This was also the case for support seeking ( $\gamma_{01}$ Non-exam. support seek $\left.=.119, p=.015\right)$, as well as AFD use as a means of coping (logistic

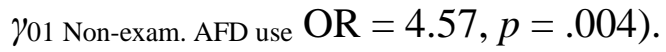




\section{Random Effects: Variability of Effects Across Participants}

To examine whether or not the correlation between actor's and partner's coping strategy use on the same day varied across couples, we estimated a random coping concordance effect. We also included a random intercept and a random previous day's own coping effect in the model, the results for which are presented Tables $2.1-2.6$ in the online supplement. A Wald test was used to get an approximate assessment of significance for the random effects.

The coping concordance effect of the partner on the actor did not vary across dyads for active coping. That is, the random coping concordance effect for same day active coping was not significant for examinees $(\tau=.002, p=.282)$ or non-examinees $(\tau=.003, p=.187)$. The effect of partner's same day support seeking varied marginally for examinees $(\tau=.006, p=.071)$, but did not vary significantly for non-examinees $(\tau=.003, p=.102)$. When the random own coping effect and the random partner effect for AFD use were entered into the model for both examinees and non-examinees, the model estimation did not converge. Thus we fit a model with only a random intercept for AFD use.

\section{Time Together}

We examined whether or not the coping concordance process may be affected by certain relationship-specific factors such as the amount of time a couple spends together. We tested the interaction between time together and the coping concordance effects for both the examinees and their mates. Consistent with the null findings in Study 1, neither of these two tests was significant. Results are presented in the online supplement, Table 2.8.

\section{Stressor Demand}

As the exam approaches, the demand associated with the stressor increases, and this produces a change in the kinds of coping strategies actors use (Iida et al., 2017). We thus thought that 
coping concordance patterns might also change over time as the bar exam neared when the examinee was the actor. Specifically this change in non-examinee-examinee concordance may occur in the week before the exam (days 28 - 34) as a function of the linear progression of time. We examined this by creating two time indicators, one for days 2-27 (where days 28 - 34 were coded as 0 and days $2-27$ were coded as $1-26$ ) and the other for days 28-34 (where days $2-$ 27 were coded as 0 and days $28-34$ were coded as $1-7)$. We found no interaction with either time measure for examinee's or non-examines active coping or AFD use. However, support seeking concordance diminished as time progressed in the last week for both examinees $\left(\gamma_{20}\right.$ Exam. support seek $=-.026, t(6384)=-2.77, p=.006)$, and non-examinees $\left(\gamma_{20}\right.$ Non-exam. support seek $=-.029$, $t(6339)=-3.13, p=.002)$, such that as the exam drew closer, both examinees and non-examinees were less likely to ask for support if their partners also sought support. The support seeking concordance effect varied significantly from time 1 to time 2 for both examinees $(t(6384)=2.67$, $p=.008)$, and non-examinees, $(t(6338)=2.92, p=.003)$. Results are presented in the online supplement, Table 2.9.

\section{Partner's Coping Association Specificity}

As in Study 1, we hypothesized that a person's use of a coping strategy should only be correlated with the partner's use of that specific coping strategy rather than any coping strategy on a given day (i.e., same-day coping specificity). We tested 12 models in which examinee's and non-examinee's active coping, support seeking, and AFD use was predicted by the partner's different coping strategy types that same day. We also included the partner's same day use of the outcome strategy, as this should continue to predict one's use of the outcome strategy. In addition, we adjusted for own previous day's use of the outcome strategy, partner's average use of the different strategy type, and day in the study. Random effects for the intercept, lagged day 
own coping, and same day partner's outcome and different strategy type were also examined.

Examinee's active coping, support seeking, and AFD use was significantly predicted by non-examinee's use of the specific strategy only, even when adjusting for different strategies. Different strategies did not predict the specific ones of interest. A similar pattern of results emerged for non-examinees, such that examinees' use of different strategy types did not significantly predict non-examinee's specific coping behavior - only the specific coping strategies did even when adjusting for use of different strategies. A summary of examinees' and non-examinees' same day coping effects on partner's coping can be found in the online supplement, Table 2.10.

\section{Discussion}

In Study 2 we examined the dynamic process of coping concordance in couples in which both partners' coping behaviors were tracked. We found that a partner's use of active coping, support seeking, and alcohol, food, and drug use on a given day was related to the actor's own use of that coping strategy, above and beyond one's own use of the strategy the previous day and above and beyond both own and partner's general tendency to use a particular coping strategy on average (i.e., average similarity between partners). The same day partner effects suggest that the immediate situational coping behaviors of a partner on a given day correspond to one's own, and that the coping concordance process may be a dynamic one.

The coping concordance effect emerged in both examinees and non-examinees as predicted, but we did find variations in these patterns for support seeking as the bar exam approached. Both non-examinees and examinees sought less support on the week prior to the exam if their partners sought more support. This was not the case during the first 3 weeks of the study, when the stressor was presumably less demanding. 
As in Study 1, coping concordance was not moderated by the amount of time a couple spent together. This is consistent with the fact that the partner's coping random effects were not significant, which suggests that coping concordance was relatively stable across persons.

Unlike the results in Study 1, however, we found that coping concordance is specific to a particular coping strategy type - that is, the association between one's own and one's partner's coping is evident only for the same coping strategy when taking into account a partner's use of the outcome strategy. Because in Study 1 we were only able to assess average, dispositional effects of coping which may be correlated with other coping strategies as well, the fact that day to day variations in coping were associated with a partner's use of the same strategy suggests that the way such an association is measured (on average or tracking daily variations) matters. In addition, the fact that we only had reports of perceived, rather than actual partner's coping in Study 1 may have also contributed to the different specificity findings.

Although the data in Study 2 show that patterns of coping within dyads are correlated, we did not test a causal relation. This result reminds us to consider other mechanisms by which daily coping might be correlated, such as dyadic level coping decisions that are not driven by one partner.

The current study allowed us to address both average coping similarity as well as daily fluctuations in coping concordance over time. In addition, we obtained varying results between examinees and partners as well as between different coping strategy types. In Study 3, we sought to replicate our results in another sample of couples without the examinee-non-examinee role differential.

\section{Study 3}

In Study 3, we made use of another longitudinal diary study of couples (Stadler, Snyder, 
Horn, Shrout, \& Bolger, 2012) to replicate the coping concordance effect found in Study 2 and extend these findings by demonstrating it in a sample of couples in which both partners were coping with daily hassles. Participants completed a background questionnaire and then filled out diaries for 35 days in which they indicated how they coped with challenges each day.

As in Study 2 we expected participants to use a specific coping strategy on a given day if their partners used that same strategy. We expected this pattern regardless of their own and their partner's average use of that strategy over the course of the study, and regardless of their own use of that strategy the previous day. However, because this sample did not exhibit a role differential - both partners were dealing with everyday stressors - we expected the coping concordance effect to be unaffected by time progression of the study. We also predicted that coping concordance between partners would be specific to a given coping strategy.

\section{Methods}

\section{Participants and Design}

Participants. Romantic couples from the New York metropolitan area were recruited for the study via fliers and postings on Internet classified sites. Eligibility criteria were that both partners were native English speakers, in a committed heterosexual relationship, had been cohabiting for at least six months at the beginning of the study, had high-speed internet access, a working email address, and were sure that they would be able to fill out the daily online diary for the following 35 days. After initial contact, both partners were screened through phone interviews for these criteria. All interested couples fulfilled eligibility criteria, except for two homosexual couples that were not included in this study.

The final sample of eligible participants who agreed to participate in the study consisted of 83 heterosexual couples (both partners had to have completed at least seven consecutive daily 
diary entries). On average, participants were 31.48 years, $(S D=9.69)$. Ethnically participants identified themselves as 54.8\% Caucasian, 13.9\% Hispanic, 16.9\% African American, 12\% Asian, and 1.8\% “other." On average, couples had been romantically involved for 6.35 years (SD $=7.25), 42 \%$ of which were married.

Procedure. Following selection for participation, data were collected in two parts. Couples were initially sent links to an online background questionnaire along with a unique identification number anonymizing their data. Then, for 35 days, both partners in each couple received daily email reminders to complete an online evening diary within one hour of going to bed. Individuals were instructed to complete the diaries independently of their partners and not to share or discuss their answers with their partner. They were also instructed to not complete any diary entries retroactively, but rather skip an entry instead of attempting to recall their mood and support events from an earlier day. The daily diaries were designed to take approximately 5-10 minutes to complete and included comparable questions as to those asked in Study 2.

\section{Measures}

The same measures were used in Study 3 as in Study 2 including time spent together (averaged between partners; women and men's report $r=.89$ ), and daily coping (measured at each day in the study) in response to the most stressful event that occurred each day. Diary days $2-35$ were included in the analyses and days were centered on day 2.

\section{Results}

\section{Coping Strategy Use Levels}

The means and standard deviations for active coping, support seeking, and AFD use are in Table 5. As in Study 2, men and women used active coping the most, and used AFD the least. On average, men used active coping and AFD as frequently as women (active coping $t(82)=.31$, 
$p=.756$; AFD $t(82)=-.85, p=.397)$, but women sought support more frequently than men $(t(82)=-2.91, p=.005)$.

\section{Coping Concordance}

To assess coping concordance in couples we again used linear multilevel models as in Study 2, but there were several important differences in the models we used. In contrast to the model in Study 2, which distinguished dyad members by whether they were examinees or nonexaminees, the models for Study 3 distinguished dyad members by gender. Also in contrast to Study 2, which fit separate models for examinees and non-examinees, the models for Study 3 were fit at the dyadic level. Because we did not expect to see - and indeed did not find differences in coping concordance patterns between men and women (see "Gender Effects" in the online supplement), we averaged the fixed lagged day effects and partner's same day effects across genders. Whereas in Study 2, we estimated six models (three for each coping strategy for each of the two actor roles), in Study 3 we only estimated three models. That is, one model was run for each coping strategy type that included both men and women. Equation 5 shows the level 1 model that was used to assess an individual's use of a particular coping strategy on a given day:

$$
A_{i t}=b_{0 M i}+b_{0 F i}+b_{1 i}\left(A_{i t-1}-A_{i} \cdot\right)+b_{2 i}\left(P_{i t}-P_{i} \cdot\right)+b_{3} D_{i t}+e_{i t}
$$

where $A_{i t}$ is use of a particular strategy for person $i$ who is the actor, on day $t ; A_{i}$. is the actor's mean use of a particular coping strategy over 34 days; $A_{i t-1}-A_{i}$. is an actor's own person-centered use of the coping strategy on day $t-1 ; P_{i t}-P_{i}$. is the person-centered partner's use of a coping strategy on day $t$ for a given actor; $D_{i t}$ is the time progression through the study on day $t$ centered on day 2, the starting day of the days analyzed; $e_{i t}$ is the residual of individual $i$ on day $t$. The coefficients $b_{O M i}$ and $b_{O F i}$ represent the intercept of a particular coping strategy's use for males, 
$M$, and females, $F$, when all independent variables are at their average on day 2 . Each of these coefficients can be viewed as the sum of a fixed effect (the overall male or female mean) and a random effect, reflecting the dispositional variation of males or females in using the target coping strategy. The correlation of these random effects reflects the dispositional coping effects that were reported in Study 2 as regression coefficients.

The coefficient $b_{1 i}$ in Equation 5 represents the effect of the individual's own personcentered coping on day $t-1$ on his or her coping on day $t$. The coefficient $b_{2 i}$ represents the effects of partner's person-centered coping on day $t$ on the individual's coping. We estimated the coping concordance effect at the level of the dyad, rather than separately for males and females. That is, we averaged across male and female partner effects. The dyad-level coping concordance effect is represented by the following equation:

$$
b_{2 i}=\gamma_{20}+u_{2 i}
$$

Coefficient $b_{3}$ in Equation 5 is the effect of time in days on the individual's use of a particular coping strategy. As in Study 2, we adjusted for own coping lagged day residuals using an autoregressive covariance structure for the active coping and support seeking models. The same-day partner's coping effect denoted as $\gamma_{20}$, is the effect of a partner's use of a coping strategy on the individual's use of that strategy on the day when the partner engages in a particular coping strategy more compared to the partner's average use of that strategy.

\section{Fixed Effects: Average Regression Effects}

Partner's same day coping. As in Study 2, the individual's use of active coping, support seeking, and AFD was associated with the partner's use of that particular coping strategy the same day (Table 6). Individuals were more likely to use active coping on a given day if their partners used active coping more than they typically do, on that same day $\left(\gamma_{20}\right.$ active coping $=.061, p$ 
$=.016)$. Similarly, individuals were more likely to seek support if partners sought more support on a given day than they typically do, $\left(\gamma_{20}\right.$ support seek $\left.=.112, p<.001\right)$. Finally, individual's AFD use on a given day was positively associated with partner's AFD use that day (logistic $\gamma_{20}$ AFD use $=.706, \mathrm{OR}=2.03, p<.001)$ as well. The full models are presented in the online supplement, Tables $3.1-3.3$.

In the fourth column of Table 6 we report the correlation between male and female random intercepts for each coping strategy; these are analogous to the dispositional coping partner effects in Study 2. As in Study 2, we found that a partner's active coping $(r=.628, p<$ $.001)$ and AFD use $(r=.536, p=.006)$ were significantly associated with the other partner's use of the same strategy on average. The association between partners' average support seeking was marginally significant $(r=.268, p=0.054)$, but positive and consistent with the results of Study 2.

As in Study 2, we also examined partner's same day effect on one's own coping without adjusting for one's own lagged day coping. A partner's use of active coping and support seeking continued to significantly predict the partner's use of the same strategy when we did not adjust for one's own coping the previous day. AFD use became marginally significant, but the effect was positive and consistent with the previous findings. Results can be found in the online supplement, Table 3.4 .

\section{Random Effects: Variability of Effects Across Participants}

The last column of Table 6 shows that the magnitude of the same day coping effects varied across couples for two of the three coping strategies. The random effect for the partner effect on same day active coping was significant for individuals (averaged for men and women, $\tau$ $=.025, p<.001)$, as was the random effect for partners' same day support seeking $(\tau=.027, p<$ 
.001). As in Study 2, the addition of the random own coping effect and the random partner effect prevented the model for AFD use from converging. Thus, only the random intercept was included in the model. Results for random intercepts and random own coping effects are presented in Tables $3.1-3.3$ of the online supplement.

\section{Time Together}

As in Study 2, we examined the amount of time a couple spent together on a given day as a moderator of coping concordance. While time together did not moderate the effect of partner's coping on one's active coping or AFD use, a significant interaction between partner's support seeking and time together did emerge $\left(\gamma_{20}\right.$ support seek $\left.=.013, t(3408)=3.36, p<.001\right)$, such that the effect of partner's support seeking on one's own support seeking increased as time spent with the partner increased. Results are presented in the online supplement, Table 3.5

\section{Partner's Coping Association Specificity}

As in Study 2, we assessed whether or not active coping, support seeking, and AFD use was predicted by partner's different coping strategy types that same day to replicate our coping association specificity findings. We included the individual's own previous day's use of the same strategy and adjusted for day in the study. We also included a random intercept and random own lagged day as well as same day partner effects for the continuous variable models.

The specificity results for active coping were mixed. Whereas active coping was not significantly predicted by partner's same day AFD use or partner's support seeking, the association of partner's active coping was diminished when adjusted for these non-specific partner coping behaviors. The association of partner's active coping was only a trend when adjusted for AFD use $\left(\gamma_{20}\right.$ active coping $\left.=.044, t(82)=1.74, p=.085\right)$ and it was non-significant when adjusted for support seeking $\left(\gamma_{20}\right.$ active coping $\left.=.037, t(82)=1.45, p=.151\right)$. There was more 
specificity for support seeking. This coping behavior was not predicted by either partner's active coping or AFD use and partner's support seeking continued to predict one's own support seeking when adjusting for partner's active coping, $\left(\gamma_{20 \text { support seek }}=.105, t(78)=4.11, p<.001\right)$, and AFD use, $\left(\gamma_{20}\right.$ support seek $\left.=.107, t(80)=4.24, p<.001\right)$. Finally, AFD use was not predicted by partner's active coping, but partner's support seeking did have a marginal effect on one's AFD use $\left(\operatorname{logistic} \gamma_{20}\right.$ AFD use $\left.=.478, \mathrm{OR}=1.62, t(3652)=1.73, p=.084\right)$. Partner's AFD use continued to predict own AFD use (logistic $\gamma_{20}$ AFD use $\left.=.687, \mathrm{OR}=1.99, t(3652)=3.23, p=.001\right)$, but was not a stronger predictor of AFD use than partner's support seeking, $t(3652)=.58, p=.559$. The results can be found in the online supplement, Table 3.6.

\section{Discussion}

In Study 3 we replicated our coping concordance findings from Study 2 in a sample of couples without specific role differentials in which both partners were coping with daily hassles. Unlike in Study 2 we found that support seeking concordance increased as time spent together increased. However, it's important to note that this finding was not evident in Studies 1 or 2. In addition, we found that partner's support seeking was associated with one's AFD use and led to a reduction in the effect of partner's active coping on one's own active coping when included in the model. Further, we also found that unlike in Study 2, individuals varied in the extent to which their support seeking and active coping was associated with their partners' support seeking and active coping. It may be that coping concordance is a more stable process across individuals when partners are dealing with specific and chronic stressors (e.g., the bar exam). However, such processes may vary more when stressors vary across couples (i.e., daily hassles in Study 3).

\section{General Discussion}

Across three studies the current research tested the notion that romantic partners tend to 
use the same coping strategy types. Unlike previous research, which has typically examined coping similarity at the dispositional level, we sought to test whether individuals' coping behaviors are associated with their partners' coping strategies day to day. As predicted, both perceived (Study 1) and actual (Studies 2 and 3) partner's use of active coping, support seeking, and alcohol, food, and drug use was positively associated with an individual's use of the same strategy on a given day (Study 1), and across time (Studies 2 and 3). Importantly, across the two longitudinal studies of couples, we found that partners' coping strategy use was correlated on a given day, above and beyond their own and their partner's average use of that coping strategy that is, beyond any general coping similarity that may exist between partners on average (Studies 2 and 3$)$.

In addition to these overall effects, we found that some variation in coping concordance exists between couples. Random effect analyses showed that individuals varied in the extent to which their partners' active coping and support seeking was correlated with their own when partners were not differentiated based on stressor (Study 3). We also found that support seeking concordance decreased in the week prior to the bar exam when stress levels were highest (Study 2). Spending more time together was associated with increased support seeking concordance in couples who were not differentiated based on stressor (Study 3), but we did not find that time together affected similarity or concordance in Studies 1 or 2.

Our findings offered mixed support for our hypothesis that one's romantic partner's use of a given coping strategy is associated with the extent to which one will use the same coping strategy on a given day. Although there were clear specificity findings in Study 2, results in Studies 1 and 3 did not definitively show specificity. In addition to the tendency for specific coping strategies to be correlated, there may be a general correlation between the amount of 
overall coping that goes on from day to day.

\section{Implications}

According to the 2010 U.S. Census, $48.4 \%$ of US households are occupied by married heterosexual couples and $6.6 \%$ of the remaining households are occupied by non-married intimate partners of the same or opposite sex. Previous work on dyadic coping (Bodenmann, 1995, 1997, 2005) recognized the importance of the dyadic context and has examined how individual stressors can become dyadic coping events. Our findings show that partners often display coping concordance, even when the stressors they are dealing with are framed as being individual. The choice of which coping strategy one person pursues often depends in part on his/her partner's strategy use and this may occur regardless of explicit coordination between partners or specific concern about the relationship, as may arise in dyadic coping (e.g., Bodenmann, 2005). Even though the current research focused solely on if and when coping concordance between partners will emerge, viewed in light of research on dyadic coping, the current findings have important implications for relationship outcomes that should be addressed in future research. Because the kind of coordinated coping that occurs when individuals engage in dyadic coping has been shown to affect relationship outcomes (e.g., relationship quality, Falconier, Jackson, Hilpert, \& Bodenmann, 2015) we suspect that coping concordance - even when not explicitly coordinated as in dyadic coping, and that may emerge based on a less active process - may also contribute to relationship satisfaction.

It is important to consider also that because some coping strategies are known to be more effective than others at alleviating distress and promoting long term adjustment to stress, if a partner happens to influence the kind of coping strategies individuals employ (though our research does not speak to partner's influence directly), this may promote either effective coping, 
or coping that is in fact maladaptive and detrimental. Indeed, given that the effectiveness of certain coping strategies depends on the kind of stressor an individual encounters (lida et al., 2017), the fact that an individual is likely to come to adopt the coping strategy of his or her partner regardless of the nature of the stressor (e.g., chronic versus not), suggests that this may lead to poorer adjustment to stress in both partners under certain circumstances. This implies that there may be instances when it may be desirable to regulate or even avoid such partner influence. On the other hand, the adoption of more proactive and effective coping strategies that one's partner uses may help the individual deal with stressors in a more constructive manner, leading to better long term adjustment to stress. Finding ways to promote such positive partner influence may be particularly beneficial in such instances.

Further, the current findings also speak to the work on coping similarity and congruence. As noted, coping similarity has largely been addressed at the dispositional level. By taking into account both general coping style similarity between partners and day-to-day fluctuations in the association between partners' coping, we gain a more nuanced perspective of the association between partners' coping. We can then begin to develop more precise hypotheses regarding when average coping similarity and day-to-day fluctuations in coping concordance will come to impact outcomes.

\section{Limitations and Future Directions}

Although we found consistent findings of coping concordance over one cross sectional study and two diary studies, we did not identify the causal process that induced the concordance with the present data. The cross-sectional design of Study 1 did not allow strong inference about causal process, and the two diary study designs did not allow us to determine if the individual's coping behavior led or co-occurred with the partner's coping behavior. An important future 
direction should be to examine fine-grained sequences of coping behavior and reports of reasons for selection of specific coping mechanisms as they occur. It would also be interesting to determine the extent to which the similar findings of Studies 2 and 3 are due to the nature of the dyadic samples of young couples living in a dense urban region.

Future studies should also examine the explicit coping goals of the partners to determine the extent to which the goals are framed as dyadic versus individual. For instance, coping with the stressor of the upcoming bar exam (Study 2) may be framed as an individual coping goal the examinee is concerned and may be engaging in active coping by studying regularly to deal with the stressor, whereas the non-examinee is not particularly involved in pursuit of this coping goal. However, we find that the vast majority of non-examinees reported concern about their partners' upcoming exam and many of their stressors were exam-related. In this case, the coping goal may have been a dyadic one and under such circumstances it is feasible that coping efforts become better-coordinated.

In addition, because Studies 2 and 3 involved secondary analysis of data that had been collected to study support processes, we were limited by the detail on coping obtained by the original investigators. In particular, we note that the composites of coping strategy types were unbalanced. For instance, active coping consisted of an average of 5 coping strategies, whereas alcohol, food, and drug use was a single item measure. The amount of variation in the latter coping measure was limited relative to the active coping measure and we needed to switch to a logistic model to analyze the variation. Nonetheless, the pattern of results regarding coping concordance across the three domains was similar.

It seems feasible that the behavioral concordance we found may extend to goals other than stress reduction. There is ample evidence that romantic partners influence each other's 
individual goal pursuits (e.g., Finkel \& Fitzsimons, 2011; Fitzsimons, Finkel, \& vanDellen, 2015). Concordance of goal-pursuit behavior similar to coping concordance might exist among members of couples who are invested in each other's individual goals and this would be interesting to examine as well.

Additional work is also needed to understand the impact of larger patterns of change during a stress process such as the bar exam preparation. We adjusted for constant dispositional tendencies in our analyses, but Curran and Bauer (2011) encourage researchers to consider investigating mechanisms that involve trajectories of patterns in addition to day-to-day processes.

\section{Concluding Comments}

Coping with individual stressors can be a social process as well as an individual selfregulation process. This is true for both adaptive and maladaptive coping behaviors. We found coping concordance on the average for three classes of coping behaviors, but we also found that the strength of the concordance varied over couples. Although we focused on concordance within the context of intimate partners, it is easy to imagine that similar processes would occur in family, roommate, and work group social contexts. 


\section{References}

Bargiel-Matusiewicz, K., \& Omar, H. A. (2015). Factors facilitating constructive coping with stress. International Journal of Child and Adolescent Health, 8(1), 41-47.

Ben-Zur, H., Gilbar, O., \& Lev, S. (2001). Coping with breast cancer: patient, spouse, and dyad models. Psychosomatic Medicine, 63(1), 32-39.

Bigatti, S. M., Brown, L. F., Steiner, J. L., \& Miller, K. D. (2011). Breast cancer in a wife: how husbands cope and how well it works. Cancer Nursing, 34(3), 193-201.

Bodenmann, G. (1995). A systemic-transactional conceptualization of stress and coping in couples. Swiss Journal of Psychology / Schweizerische Zeitschrift Fur Psychologie / Revue Suisse De Psychologie, 54(1), 34-49.

Bodenmann, G. (1997). Dyadic coping: A systemic-transactional view of stress and coping among couples: Theory and empirical findings. European Review of Applied Psychology / Revue Europeenne De Psychologie Appliquee, 47(2), 137-141.

Bodenmann, G. (2005). Chapter: Dyadic coping and its significance for marital functioning. In T. A. Revenson, K. Kayser \& G. Bodenmann (Eds.), Couples coping with stress: Emerging perspectives on dyadic coping (pp. 33-49). Washington, DC: American Psychological Association.

Bouchard, G., Sabourin, S., Lussier, Y., Wright, J., \& Richer, C. (1998). Predictive validity of coping strategies on marital satisfaction: Cross-sectional and longitudinal evidence. Journal of Family Psychology, 12(1), 112-131.

Carver, C. S., \& Connor-Smith, J. (2010). Personality and coping. Annual Review of Psychology, $61,679-704$.

Carver, C. S., Scheier, M. F., \& Weintraub, J. K. (1989). Assessing coping strategies: A 
theoretically based approach. . Journal of Personality and Social Psychology, 56(2). 267283.

Curran, P. J., \& Bauer, D. J. (2011). The disaggregation of within-person and between-person effects in longitudinal models of change. Annual review of psychology, 62, 583-619.

Dahlquist, L. M., Czyzewski, D. I., Copeland, K. G., Jones, C. L., Taub, E., \& Vaughan, J. K. (1993). Parents of children newly diagnosed with cancer: Anxiety, coping, and marital distress. Journal of Pediatric Psychology, 18(3), 365-376.

Eckenrode, J., (2013). The social context of coping. New York: Springer.

Falconier, M. K., Jackson, J. B., Hilpert, P., \& Bodenmann, G. (2015). Dyadic coping and relationship satisfaction: A meta-analysis. Clinical Psychology Review, 42, 28-46.

Finkel, E. J., \& Fitzsimons, G. M. (2011). The effects of social relationships on self-regulation. In K. D. Vohs, \& R. F. Baumeister (Eds.), Handbook of self-regulation: Research, theory, and applications (2nd ed., pp 390-406). New York: Guilford Press.

Fitzsimons, G. M., Finkel, E. J., \& vanDellen, M. R. (2015). Transactive goal dynamics. Psychological Review. 122, 648-673.

Folkman, S., \& Lazarus, R. S. (1988). Coping as a mediator of emotion. Journal of Personality and Social Psychology, 54(3), 466-475.

Folkman, S., \& Moskowitz, J. T. (2000). Stress, positive emotion, and coping. Current Directions in Psychological Science, 9(4), 115-118.

Giunta, C. T., \& Compas, B. E. (1993). Coping in marital dyads: Patterns and associations with psychological symptoms. Journal of Marriage and the Family, 55(4), 1011-1017.

Gleason, M. E. J., Iida, M., Bolger, N., \& Shrout, P. (2003). Supportive equity in close relationships. Personality and Social Psychology Bulletin, 29, 1036-1045. 
Gleason, M. E. J., Iida, M., Shrout, P. E., \& Bolger, N. (2008). Receiving support as a mixed blessing: Evidence for dual effects of support on psychological outcomes. Journal of Personality and Social Psychology, 94(5), 824-838.

Holahan, C. J., Moos, R. H., Moerkbak, M. L., Cronkite, R. C., Holahan, C. K., \& Kenney, B. A. (2007). Spousal similarity in coping and depressive symptoms over 10 years. Journal of Family Psychology : JFP : Journal of the Division of Family Psychology of the American Psychological Association (Division 43), 21(4), 551-559.

Iida, M., Green-Rapaport, A.S., Gleason, M., Bolger, N., Shrout, P.E., (2017). Daily coping choices and the influence of daily coping on anxiety under examination stress: A model of interindividual differences in intraindividual change. Personality and Social Psychology Bulletin. 43(7), 907-923.

Iida, M., Seidman, G., Shrout, P. E., Fujita, K., \& Bolger, N. (2008). Modeling support provision in intimate relationships. Journal of Personality and Social Psychology, 94, 460-478.

Kenny, D. A., \& Acitelli, L. K. (2001). Accuracy and bias in the perception of the partner in a close relationship. Journal of personality and social psychology, 80(3), 439-448.

Lazarus, R. S., \& Folkman, S. (1984). Stress, appraisal, and coping. New York: Springer.

Monnier, J., Cameron, R. P., Hobfall, S. E., \& Gribble, J. R. (2000). Direct and crossover effects of prosocial and antisocial coping behaviors. Journal of Family Psychology, 14(4), 570584.

Rabe-Hesketh, S., Skrondal, A., \& Pickles, A. (2004). Generalized multilevel structural equation modeling. Psychometrika, 69(2), 167-190.

Revenson, T. A. (2003). Scenes from a marriage: Examining support, coping, and gender within the context of chronic Illness. Social psychological foundations of health and illness. In J. 
Suls, \& K. A. Wallston, (Eds.). Malden: Blackwell Publishing Ltd.

Shrout, P.E., Bolger, N., Iida, M., Burke, C., Gleason, M.E.J., \& Lane, S.P. (2010). The Effects of Daily Support Transactions During Acute Stress: Results From a Diary Study of Bar Exam Preparation. In K. T. Sullivan and J. Davila (Eds.), Support Processes in Intimate Relationships (pp. 175-199), New York: Oxford University Press.

Shrout, P. E. (2014). Why do study participants report fewer symptoms and problems in later waves of longitudinal studies? Presentation at the 142nd Annual Meeting of the American Public Health Association, November 18, 2014, New Orleans, LA.

Sirois, F. M., Molnar, D. S., \& Hirsch, J. K. (2015). Self-compassion, stress, and coping in the context of chronic illness. Self and Identity, 14(3), 334-347.

Skinner, E. A., Edge, K., Altman, J., \& Sherwood, H. (2003). Searching for the structure of coping: A review and critique of category systems for classifying ways of coping. Psychological Bulletin, 129(2), 216-269.

Solberg Nes, L., \& Segerstrom, S. C. (2008). Conceptualizing coping: Optimism as a case study. Social and Personality Psychology Compass, 2(6), 2125-2140.

Stadler, G., Snyder, K.A., Horn, A.B., Shrout, P.E., \& Bolger, N.P. (2012). Close relationships and health in daily life: A review and empirical data on intimacy and somatic symptoms. Psychosomatic Medicine, 74, 398-409.

Todd, M., Tennen, H., Carney, M. A., Armeli, S., \& Affleck, G. (2004). Do we know how we cope? Relating daily coping reports to global and time-limited retrospective assessments. Journal of Personality and Social Psychology, 86(2), 310-319.

U. S. Census Bureau. (2010). Households and families: 2010, 2010 Census briefs. Retrieved from https://www.census.gov/prod/cen2010/briefs/c2010br-14.pdf 
Wamboldt, F. S., Steinglass, P., \& De-Nour, A. (1991). Coping within couples: adjustment two years after forced geographic relocation. Family Process, 30(3), 347-361.

Watson, D., Klohnen, E. C., Casillas, A., Nus Simms, E., Haig, J., \& Berry, D. S. (2004). Match makers and deal breakers: Analyses of assortative mating in newlywed couples. Journal of Personality, 72, $1029-1068$.

Zimmer-Gembeck, M. J., \& Skinner, E. A. (2011). Review: The development of coping across childhood and adolescence: An integrative review and critique of research. International Journal of Behavioral Development, 35(1), 1-17. 


\section{Footnotes}

1. Study 2 consists of secondary analyses. The coping variables examined in the current work have also been examined by Iida, Green-Rapaport, Gleason, Bolger and Shrout (2017) as well as by Iida, Seidman, Shrout, Fujita, and Bolger (2008). However, none of the previous work has examined associations between partners' coping.

2. We excluded the first day of the study from our analyses because it has been shown that initial responses decrease substantially following the first day of a diary study (Shrout, 2014).

3. An alternate analysis would be to combine examinees and partners into a dyadic model that estimated both coping concordance processes simultaneously. We investigated this alternative approach, but found that the estimation would not converge. We speculate that this is due to the very different coping demands of the examinees and partners would lead to very different covariance patterns in both the independent variables and the outcomes.

4. To adjust for temporal dependency, various approaches may be used that may have implications for fixed effects. Specifically, adjusting for the lagged variable residuals changed the sign of the lagged fixed effect coefficient. However, the fixed effect of interest - partner's coping on a given day - remains consistent across models with different covariance structures of the residuals, as well as models that do not adjust for such temporal dependency. 
Table 1

Study 1: Descriptive statistics and correlations of key variables.

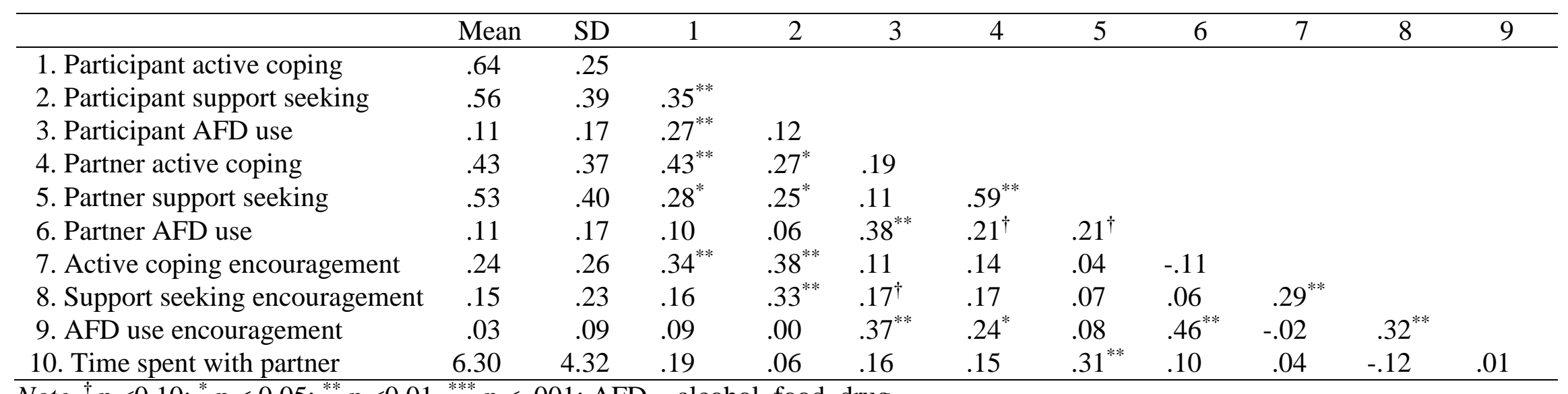

Note. ${ }^{\dagger} p<0.10 ;{ }^{*} p<0.05 ;{ }^{* *} p<0.01,{ }^{* * *} p<.001 ; \mathrm{AFD}=$ alcohol, food, drug 
Table 2

Study 1: Ordinary least squares regression analyses predicting participant's coping from partner's coping and partner's encouragement to cope

\begin{tabular}{lll}
\hline \multicolumn{1}{c}{ Predictor } & \multicolumn{1}{c}{$b(S E)$} & $95 \% \mathrm{CI}$ \\
\hline Active coping & $.266(.069)^{* * *}$ & $(.13, .40)$ \\
Partner's active coping & $.244(.097)^{*}$ & $(.05, .44)$ \\
Partner's encouraged coping & .252 & \\
$\mathrm{R}^{2}$ for active coping & & \\
Support seeking & $.206(.099)^{*}$ & $(.01, .40)$ \\
Partner's support seeking & $.470(.166)^{* *}$ & $(.14, .80)$ \\
Partner's encouraged coping & .155 & \\
$\mathrm{R}^{2}$ for support seeking & $.192(.112)^{\dagger}$ & $(-.03, .42)$ \\
AFD use & $.757(.216)^{* * *}$ & $(.33,1.19)$ \\
$\begin{array}{l}\text { Partner's AFD use } \\
\text { Partner's encouraged coping }\end{array}$ & .299 & \\
$\mathrm{R}^{2}$ for AFD use & ${ }^{* * * *} p<.001 ;$ AFD = alcohol, food, drug \\
\hline Note ${ }^{\dagger} p<0.10 ;{ }^{*} p<0.05 ;{ }^{* *} p<0.01$, &
\end{tabular}


Table 3

Study 2: Descriptive statistics for coping strategy type use by examinees and their non-examinee partners and for time spent together.

The correlations above the diagonal are computed on day-to-day variations in coping and time spent together, and the correlations below the diagonal are computed using person-level (dispositional) averages of coping and reported time spent together.

\begin{tabular}{|c|c|c|c|c|c|c|c|c|c|}
\hline & Mean & SD & 1 & 2 & 3 & 4 & 5 & 6 & 7 \\
\hline 1. Examinee's active coping & .32 & .28 & & $.11^{* *}$ & 0 & $.05^{* *}$ & -.01 & -.02 & -.10 \\
\hline 2. Examinee's support seeking & .18 & .28 & $.47^{* *}$ & & $.08^{* *}$ & -.01 & $.03^{*}$ & $.02^{\dagger}$ & $.04^{* *}$ \\
\hline 3. Examinee's AFD use & .16 & .36 & .02 & $.10^{\dagger}$ & & -.02 & 0 & $.04^{* *}$ & $-.04^{* *}$ \\
\hline 4. Non-examinee's active coping & .27 & .27 & $.16^{* *}$ & $.21^{* *}$ & .06 & & $.14^{* *}$ & -.01 & $-.07^{* *}$ \\
\hline 5. Non-examinee's support seeking & .15 & .25 & $.12^{*}$ & $.13^{*}$ & 0 & $.57^{* *}$ & & $.08^{* *}$ & $-.02^{*}$ \\
\hline 6. Non-examinee's AFD use & .09 & .28 & 0 & .10 & $.17^{* *}$ & $.19^{* *}$ & $.34^{* *}$ & & $-.02^{*}$ \\
\hline 7. Time spent together & 4.52 & 3.68 & .03 & .09 & $-.13^{*}$ & $.12^{*}$ & $.20^{* *}$ & 0 & \\
\hline
\end{tabular}

Note. ${ }^{\dagger} p<0.10 ;{ }^{*} p<0.05 ;{ }^{* *} p<0.01,{ }^{* * *} p<.001 ; \mathrm{AFD}=$ alcohol, food, drug


Table 4

Study 2: Same day unique coping $\left(\gamma_{20}\right)$ and dispositional coping $\left(\gamma_{01}\right)$ effects of partner's coping on own coping on a given day, adjusted for own lagged day coping (person-centered), and day in the study (centered on day 2). Results are from six separate analyses, one for each coping outcome. Estimates for active coping and support seeking are from linear mixed models, whereas estimates for AFD are from logistic (generalized) mixed models.

\begin{tabular}{|c|c|c|c|}
\hline Outcome & Estimate $(S E)$ & $95 \% \mathrm{CI}$ & Random Effect $(S E)$ \\
\hline \multicolumn{4}{|l|}{ Examinee's active coping } \\
\hline Same day unique coping $\left(\gamma_{20}\right)$ & $.053(.012)^{* * *}$ & $(.03, .08)$ & $.002(.003)$ \\
\hline Dispositional coping $\left(\gamma_{01}\right)$ & $.172(.063)^{* *}$ & $(.05, .30)$ & \\
\hline \multicolumn{4}{|l|}{ Examinee's support seeking } \\
\hline Same day unique coping $\left(\gamma_{20}\right)$ & $.046(.014)^{* * *}$ & $(.02, .07)$ & $.006(.006)^{\dagger}$ \\
\hline Dispositional coping $\left(\gamma_{01}\right)$ & $.165(.069)^{*}$ & $(.03, .30)$ & \\
\hline \multicolumn{4}{|l|}{ Examinee's AFD use } \\
\hline Same day unique coping $\left(\gamma_{20}\right)$ & $.400(.156)^{*}$ & $(.07, .69)$ & $0^{\mathrm{a}}$ \\
\hline Dispositional coping $\left(\gamma_{01}\right)$ & $2.092(.710)^{* *}$ & $(.70,3.49)$ & \\
\hline \multicolumn{4}{|l|}{ Non-examinee's active coping } \\
\hline Same day unique coping $\left(\gamma_{20}\right)$ & $.056(.012)^{* * * *}$ & $(.03, .08)$ & $.003(.003)$ \\
\hline Dispositional coping $\left(\gamma_{01}\right)$ & $.154(.053)^{* *}$ & $(.05, .26)$ & \\
\hline \multicolumn{4}{|l|}{ Non-examinee's support seeking } \\
\hline Same day unique coping $\left(\gamma_{20}\right)$ & $.037(.012)^{* *}$ & $(.01, .06)$ & $.003(.003)$ \\
\hline Dispositional coping $\left(\gamma_{01}\right)$ & $.119(.049)^{*}$ & $(.02, .22)$ & \\
\hline \multicolumn{4}{|l|}{ Non-examinee's AFD use } \\
\hline Same day unique coping $\left(\gamma_{20}\right)$ & $.360(.154)^{*}$ & $(.06, .66)$ & $0^{\mathrm{a}}$ \\
\hline Dispositional coping $\left(\gamma_{01}\right)$ & $1.517(.528)^{* *}$ & $(.48,2.55)$ & \\
\hline \multicolumn{4}{|c|}{ Note. ${ }^{\dagger} p<0.10 ;{ }^{*} p<0.05 ;{ }^{* *} p<0.01,{ }^{* * *} p<.001 ;$ AFD = alcohol, food, drug } \\
\hline
\end{tabular}


Table 5

Study 3: Descriptive statistics of men's and women's coping strategy type use and time spent together, correlations computed based on day-to-day variations in coping and time spent together (top half of diagonal), and overall person-level (dispositional) correlations (bottom half of diagonal).

\begin{tabular}{|c|c|c|c|c|c|c|c|c|c|}
\hline & Mean & SD & 1 & 2 & 3 & 4 & 5 & 6 & 7 \\
\hline 1. Men's active coping & .29 & .19 & & $.24^{* *}$ & $.11^{* *}$ & $.08^{* *}$ & $.08^{* *}$ & $.05^{*}$ & 0 \\
\hline 2. Men's support seeking & .14 & .15 & $.65^{* *}$ & & $.10^{* *}$ & $.05^{*}$ & $.15^{* *}$ & $.05^{*}$ & .02 \\
\hline 3. Men's alcohol, food, and drug use & .10 & .19 & $.20^{\dagger}$ & $.28^{*}$ & & .02 & $.08^{* *}$ & $.11^{* *}$ & 0 \\
\hline 4. Women's active coping & .29 & .18 & $.54^{* *}$ & $.36^{* *}$ & .16 & & $.21^{* *}$ & $.08^{* *}$ & -.02 \\
\hline 5. Women's support seeking & .20 & .16 & $.29^{* *}$ & $.34^{* *}$ & $.18^{\dagger}$ & $.66^{* *}$ & & $.09^{* *}$ & $.04^{*}$ \\
\hline 6. Women's alcohol, food, and drug use & .12 & .21 & .14 & .04 & $.36^{* *}$ & $.31^{* *}$ & $.54^{* *}$ & & 0 \\
\hline 7. Time spent together & 4.63 & 11.85 & $.19^{\dagger}$ & .15 & .08 & .16 & .11 & .06 & \\
\hline
\end{tabular}

Note. ${ }^{\dagger} p<0.10 ;{ }^{*} p<0.05 ;{ }^{* *} p<0.01,{ }^{* * *} p<.001 ;$ AFD = alcohol, food, drug 
Table 6

Study 3: Same day unique coping $\left(\gamma_{20}\right)$ effects on own coping on a given day, adjusted for own lagged day coping (person-centered), and day in the study (centered on day 2) as well as the correlation between male and female random intercepts. Estimates for active coping and support seeking are from linear mixed models, whereas estimates for AFD are from logistic (generalized) mixed models.

\begin{tabular}{|c|c|c|c|c|}
\hline \multirow[t]{2}{*}{ Outcome } & \multicolumn{2}{|c|}{ Same day unique coping $\left(\gamma_{20}\right)$} & \multirow{2}{*}{$\begin{array}{c}\text { Male- } \\
\text { female } \\
\text { intercept } \\
\text { correlation }\end{array}$} & \multirow{2}{*}{$\begin{array}{c}\begin{array}{c}\text { Same day } \\
\text { coping random } \\
\text { effect }(\tau)\end{array} \\
\text { Estimate }(S E)\end{array}$} \\
\hline & Estimate $(S E)$ & $95 \% \mathrm{CI}$ & & \\
\hline Active coping & $.061(.025)^{*}$ & $(.01, .11)$ & $.628^{* * *}$ & $.025(.008)^{* * * *}$ \\
\hline Support seeking & $.112(.025)^{* * * *}$ & $(.07, .17)$ & $.268^{\dagger}$ & $.027(.008)^{* * *}$ \\
\hline AFD use & $.706(.212)^{* * *}$ & $(.29,1.12)$ & $.536^{* *}$ & 0 \\
\hline
\end{tabular}

